\title{
Design, Synthesis and Evaluation of the Biological Activities of Some New Carbohydrazide and Urea Derivatives
}

\author{
Bazı Yeni Karbohidrazit ve Üre Türevlerinin Tasarımı, Sentezi ve Biyolojik \\ Aktivitelerinin Değerlendirilmesi
}

\author{
(D) Fatih TOK'1 (D) Recep ILHAN², (D) Selin GÜNAL², (D) Petek BALLAR-KIRMIZIBAYRAK², (D) Bedia KOÇYiĞiT-KAYMAKÇIOĞLU1* \\ 'Marmara University, Faculty of Pharmacy, Department of Pharmaceutical Chemistry, İstanbul, Turkey \\ ²Ege University, Faculty of Pharmacy, Department of Biochemistry, Izmir, Turkey
}

\begin{abstract}
Objectives: Urea and carbohydrazide derivatives are important compounds exhibiting cytotoxic activities. In this study, a series of new urea and carbohydrazide derivatives containing an pyridine ring were synthesized and evaluated for cytotoxic activity.

Materials and Methods: The proposed structures of the synthesized compounds were confirmed using elemental analysis, IR, and ${ }^{1} \mathrm{H}-\mathrm{NMR}$ spectroscopic techniques. The cytotoxic potencies of synthesized compounds were determined using a 3-(4,5-dimethylthiazol-2-yl)-2,5diphenyltetrazolium bromide assay (MTT) on BRCA mutant-carrying HCC1937 and Capan-1 cell lines, as well as on MCF7, HeLa, and MRC5 cells.

Results: 3a, 3b, 3c and 3d showed cytotoxic activity against all cancer cell lines.

Conclusion: Our data indicate that compounds 3a-d are more selective to cancer cells compared with nontumoral fibroblasts; however, these compounds are not more potent on HR defective cells with BRCA mutants.
\end{abstract}

Key words: Carbohydrazide, urea, cytotoxic activity

öz

Amaç: Üre ve karbohidrazit türevleri önemli sitotoksik etkinlik gösteren bileşiklerdir. Bu çalışmada, bir yeni seri piridin halkası taşıyan üre ve karbohidrazit türevleri sentezlenmiş ve sitotoksik etkileri araştırılmıştır.

Gereç ve Yöntemler: Sentezlenen bileșiklerin önerilen yapıları elemental analiz, IR, ${ }^{1} \mathrm{H}-\mathrm{NMR}$ spektroskopik yöntemleriyle doğrulanmıştır. Sentezlenen bileşiklerin sitotoksik etki güçleri, 3-(4,5-dimetiltiyazol-2-il)-2,5-difeniltetrazolyum bromür (MTT) kullanılarak BRCA mutasyonu taşıyan HCC1937 ve Capan-1 hücre hatları olan MCF7, HeLa ve MRC5 hücreleri üzerinde tespit edilmiştir.

Bulgular: 3a, 3b, 3c ve 3d tüm kanser hücre hatlarına karşı sitotoksik etki göstermiştir.

Sonuç: Verilerimiz, 3a-d bileşiklerinin kanser hücrelerinde tümöral olmayan fibroblastlarla kıyaslandığında daha seçici olduğunu, ancak bu bileşiklerin BRCA1 mutant homolog rekombinasyonal (HR) DNA onarımı hatalı hücrelerde ise daha fazla etkili olmadığını göstermektedir.

Anahtar kelimeler: Karbohidrazit, üre, sitotoksik aktivite

\section{INTRODUCTION}

Cancer is one of the major causes of death worldwide. The development of identification and treatment are important for cancer treatment. However, effective and selective treatment methods are insufficient against some types of cancer. Scientists continue their studies to find effective molecules for cancer treatment., ${ }^{1,2}$ Compounds bearing nitrogen, sulfur, and oxygen play a significant role by forming hydrogen bonds with DNA. ${ }^{3}$ Therefore heterocyclic compounds such as pyridine and pyrimidine showed anticancer, ${ }^{4}$ antibacterial, ${ }^{5}$ antifungal, ${ }^{6}$ analgesic, and anti-inflammatory activity.

Sorafenib, which carries a pyridine ring and urea group was confirmed by the United States Food and Drug Administration for the treatment of renal cell carcinoma in 2005. Sorafenib, which

*Correspondence: E-mail: bkaymakcioglu@marmara.edu.tr, Phone: +90 5325079460 ORCID-ID: orcid.org/0000-0003-0817-8236 
has a broad spectrum for anticancer therapy, inhibits some kinases such as vascular endothelial and platelet-derived growth factor. Therefore, sorafenib could be used with numerous types of cancer. Chemical structures of compounds were envisioned consisting of three parts: pyridyl moiety, a linker, and urea functional group as a pharmacophore (Scheme 1). ${ }^{8}$
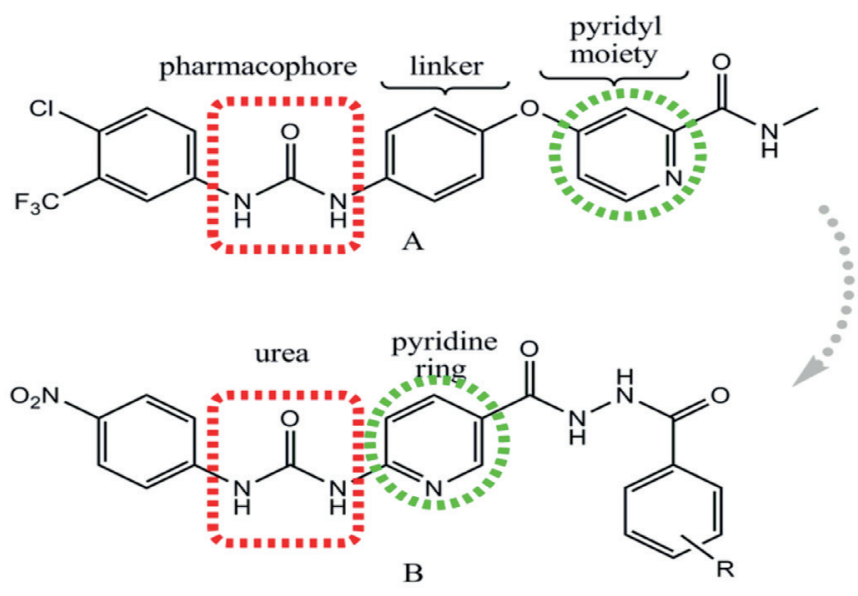

Scheme 1. The similarity of synthesized compounds (A) and sorafenib (B)

Wang et al. ${ }^{9}$ studied new benzimidazole-2-urea derivatives that decrease the proliferation of some cancer cells (HeLa, K562, HepG2) and reported that these molecules could be used as tubulin inhibitors. In another study, Fortin et al.10 discovered that compounds containing urea derivatives had greater antiproliferative activity than amide groups from their structure activity relationship. De et al. ${ }^{11}$ synthesized N'-(2(4-substitute)cyclopropanecarbonyl)isonicotinohydrazidecontaining carbohydrazide and a pyridine ring and evaluated their cytotoxic activity against A549, PC3, and U373 cells.

Pyridine is an important ring system with numerous biologic activities. For example, Kurumurthy et al. ${ }^{12}$ selected pyridine derivatives to achieve cytotoxic activity against THP1, U937, HL60 and B16-F10 cells.

Using results obtained in the literature and pharmacophore analysis (Scheme 1), target molecules carrying urea and carbohydrazide derivatives were synthesized from methyl 6-aminopyridine-3-carboxylate and evaluated their cytotoxic

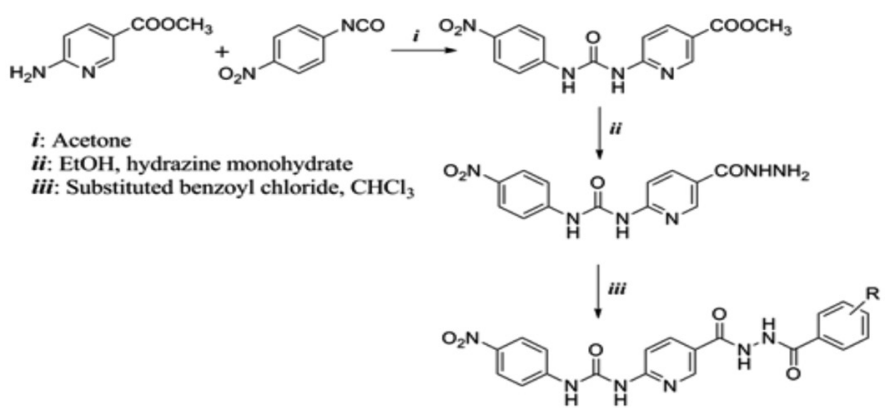

Scheme 2. The synthesis route of the compounds activity against cancer cells (HCC1937, Capan1, MCF-7, HeLa and MRC5).

\section{MATERIALS AND METHODS}

\section{Chemistry}

All chemicals reagents and solvents were obtained from Sigma-Aldrich (St. Louis, MO, USA), and Merck (Darmstadt, Germany). The homogeneity and purity of the compounds were checked using thin-layer chromatography (TLC), performed on commercially available silica gel (Kieselgel 60, F254) coated aluminum sheets (Merck) by using petroleum ether:ethyl acetate $(10: 90 \mathrm{v} / \mathrm{v})$ as the solvent system. Visualization on TLC was performed using ultraviolet light $(\lambda=254 \mathrm{~nm})$ and an iodine indicator. Melting points (MP) were determined using a Schmelzpunktbestimmer SMP II. IR spectra were recorded with a Shimadzu FTIR-8400S (Japan). 'H-NMR spectra were recorded on a Bruker Avance $400 \mathrm{MHz}$ (USA) in DMSO- $\mathrm{d}_{6}$ using tetramethylsilane (TMS) as the internal reference. Chemical shifts ( $\delta$ ) were expressed in parts per million relative to TMS and the following abbreviations were used to describe the peak patterns when appropriate: s, (singlet); d, (doublet); t, (triplet); $\mathrm{m}$, (multiplet). Elemental analysis (C, $\mathrm{H}$ and $\mathrm{N}$ ) was performed on a CHNS-Thermo Scientific Flash 2000 (Waltham, MA USA).

\section{Synthesis of urea derivatives}

Methyl 6-aminopyridine-3-carboxylate was dissolved in acetone at $80^{\circ} \mathrm{C}$. Then, a solution of the corresponding equimolar isocyanate in dry acetone was added as two parts, every 30 minutes. The reaction mixture was refluxed for 6 hours. The reaction was finalized by checking with TLC and left overnight. The precipitate was filtered off, dried, and purified with acetone. ${ }^{13}$

\section{Methyl 6-(3-(4-nitrophenyl)ureido)nicotinate (1)}

Yellow solid; Yield: $70 \%$; MP: $237-239^{\circ} \mathrm{C}$; IR $\left(v_{\max } \mathrm{cm}^{-1}\right): 3365$, $3211(\mathrm{~N}-\mathrm{H}), 3080$ (=C-H stretching), 2983, $2843(\mathrm{C}-\mathrm{H}), 1708$ (urea $\mathrm{C}=\mathrm{O}$ ), 1604, 1562, 1506, 1491, $1411\left(\mathrm{C}=\mathrm{C}, \mathrm{NO}_{2}, \mathrm{~N}-\mathrm{H}\right.$ bending, C-N), 1273 (C-O), 839 (=C-H). ${ }^{1} \mathrm{H}-\mathrm{NMR}$ (DMSO- $d_{6} / \mathrm{TMS}, 400$ $\mathrm{MHz}, \delta$ in ppm): 3.83 (s, 3H, $\left.-\mathrm{OCH}_{3}\right), 7.69-8.84(\mathrm{~m}, 7 \mathrm{H}, \mathrm{Ar}-\mathrm{H})$, 9.99 (s, 1H, NH), 10.73 (s, 1H, NH). For $\mathrm{C}_{14} \mathrm{H}_{12} \mathrm{~N}_{4} \mathrm{O}_{5}$ (M.W.: 316.27 $\mathrm{g} / \mathrm{mol}$ ) calculated (\%): C:53.17, H:3.82, N:17.71. Found: C:54.46, $\mathrm{H}: 3.66, \mathrm{~N}: 17.95$.

\section{Synthesis of the hydrazide derivatives}

Methyl 6-(3-(4-nitrophenyl)ureido)nicotinate was dissolved in ethanol on a magnetic stirrer. Then, hydrazine monohydrate (1 $\mathrm{mL}$ ) was added. The reaction mixture was refluxed for 6 hours. The mixture was filtered and washed with methanol. ${ }^{14}$

1-(5-(Hydrazinecarbonyl)pyridin-2-yl)-3-(4-nitrophenyl)urea (2)

Yellow solid; Yield: 65\%; MP: $274-275^{\circ} \mathrm{C} ; \mathrm{IR}\left(v_{\max } \mathrm{cm}^{-1}\right): 3338$, $3221(\mathrm{~N}-\mathrm{H}), 3078$ (=C-H stretching), 1712 ( $\mathrm{C}=\mathrm{O}$ urea), $1678(\mathrm{C}=\mathrm{O}$ hydrazide), $1620(\mathrm{C}=\mathrm{N}), 1562,1510,1491,1431\left(\mathrm{C}=\mathrm{C}, \mathrm{NO}_{2}, \mathrm{~N}-\mathrm{H}, \mathrm{C}-\mathrm{N}\right)$, $815\left(=\mathrm{C}-\mathrm{H}\right.$ ). ${ }^{1} \mathrm{H}-\mathrm{NMR}$ (DMSO- $d_{6} / \mathrm{TMS}, 400 \mathrm{MHz}, \delta$ in ppm): 4.48 (s, 2H, $\mathrm{NH}_{2}$ ), 7.71-8.87 (m, 7H, $\left.\mathrm{Ar}-\mathrm{H}\right), 9.93$ (s, 1H, NH), 10.04 (s, 1H, $\mathrm{NH}$ ), 10.78 (s, $1 \mathrm{H}, \mathrm{NH}$ ). $\mathrm{C}_{13} \mathrm{H}_{12} \mathrm{~N}_{6} \mathrm{O}_{4}$ (M.W.: $316.27 \mathrm{~g} / \mathrm{mol}$ ) calculated (\%): C:49.37, H:3.82, N:26.57. Found: C:51.05, H:3.97, N:25.25. 
Synthesis of the carbohydrazide derivatives

To a solution of hydrazide ( $1 \mathrm{mmol}$ ) (2) and triethylamine ( $2 \mathrm{mmol}$ ) in dry $\mathrm{CH}_{2} \mathrm{Cl}_{2}(5 \mathrm{~mL})$, a solution of previously prepared benzoyl chloride (1 mmol) was added dropwise at room temperature. The reaction mixture was stirred on a magnetic stirrer for 3 hours. The precipitate was then washed with distilled water and filtered. The purity of compounds was checked with TLC. ${ }^{15}$

1-(5-(2-(4-Fluorobenzoyl)hydrazinecarbonyl)pyridin-2-yl)-3-(4nitrophenyl)urea (3a)

Yellow solid; Yield: $70 \%$; MP: $257-259^{\circ} \mathrm{C}$; IR $\left(v_{\max } \mathrm{cm}^{-1}\right)$ : 3304 , 3207, $3122(\mathrm{~N}-\mathrm{H}), 3080(=\mathrm{C}-\mathrm{H}$ stretching), $1712(\mathrm{C}=\mathrm{m}), 1612$, 1562, 1508, $1492\left(\mathrm{C}=\mathrm{C}, \mathrm{NO}_{2}, \mathrm{~N}-\mathrm{H}, \mathrm{C}-\mathrm{N}\right), 842(=\mathrm{C}-\mathrm{H}) .{ }^{1} \mathrm{H}-\mathrm{NMR}$ (DMSO- $d_{6} / \mathrm{TMS}, 400 \mathrm{MHz}, \delta$ in ppm): 7.67-8.84 (m, $11 \mathrm{H}, \mathrm{Ar}-\mathrm{H}$ ), $9.69(\mathrm{~s}, 1 \mathrm{H}, \mathrm{NH}), 10.04(\mathrm{~s}, 1 \mathrm{H}, \mathrm{NH}), 10.84(\mathrm{~s}, 2 \mathrm{H}, \mathrm{NH}) . \mathrm{C}_{20} \mathrm{H}_{15} \mathrm{FN}_{6} \mathrm{O}_{5}$ (M.W.: $438.37 \mathrm{~g} / \mathrm{mol}$ ) calculated (\%): C:54.80, H:3.45, N:19.17. Found: C:55.24, H:3.22, N:18.65.

1-(5-(2-(4-Chlorobenzoyl)hydrazinecarbonyl)pyridin-2-yl)-3-(4nitrophenyl)urea (3b)

Yellow solid; Yield: $75 \%$; MP: $202-203^{\circ} \mathrm{C}$; IR $\left(v_{\max } \mathrm{cm}^{-1}\right)$ : 3304 , 3207, $3122(\mathrm{~N}-\mathrm{H}), 3080$ (=C-H stretching), $1712(\mathrm{C}=\mathrm{O}), 1612$, 1562, 1508, 1492, $1431\left(\mathrm{C}=\mathrm{C}, \mathrm{NO}_{2}, \mathrm{~N}-\mathrm{H}, \mathrm{C}-\mathrm{N}\right), 842(=\mathrm{C}-\mathrm{H})$. ${ }^{1} \mathrm{H}-\mathrm{NMR}$ (DMSO- $d_{6} / \mathrm{TMS}, 400 \mathrm{MHz}, \delta$ in ppm): 7.71-8.85 (m, 11H, $\mathrm{Ar}-\mathrm{H}$ ), 9.98 (s, 2H, NH), 10.73 (s, 2H, NH). $\mathrm{C}_{20} \mathrm{H}_{15} \mathrm{ClN}_{6} \mathrm{O}_{5}$ (M.W.: $454.82 \mathrm{~g} / \mathrm{mol}$ ) calculated (\%): C:52.81, H:3.32, N:18.48. Found: C:52.75, H:3.48, N:18.44.

1-(5-(2-(4-Nitrobenzoyl)hydrazinecarbonyl)pyridin-2-yl)-3-(4nitrophenyl)urea (3c)

Yellow solid; Yield: $80 \%$; MP: $273-275^{\circ} \mathrm{C}$; IR $\left(v_{\max } \mathrm{cm}^{-1}\right): 3215$, $3124(\mathrm{~N}-\mathrm{H}), 3082$ (=C-H stretching), 1714 (C=O), 1612, 1564, 1510, $1492\left(\mathrm{C}=\mathrm{C}, \mathrm{NO}_{2}, \mathrm{~N}-\mathrm{H}, \mathrm{C}-\mathrm{N}\right), 846(=\mathrm{C}-\mathrm{H}) .{ }^{1} \mathrm{H}-\mathrm{NMR}$ (DMSO-d $/$ TMS, $400 \mathrm{MHz}, \delta$ in ppm): 7.68-8.85 (m, 11H, Ar-H), 9.62 (s, $1 \mathrm{H}, \mathrm{NH}$ ), 10.00 (s, $1 \mathrm{H}, \mathrm{NH}$ ), 10.74 (s, 2H, NH). $\mathrm{C}_{20} \mathrm{H}_{15} \mathrm{~N}_{7} \mathrm{O}_{7}$ (M.W.: $465.38 \mathrm{~g} / \mathrm{mol}$ ) calculated (\%): C:51.62, H:3.25, N:21.07. Found: C:52.33, H:3.12, N:20.66.

1-(5-(2-(2,6-Dichlorobenzoyl)hydrazinecarbonyl)pyridin-2-yl)-3(4-nitrophenyl)urea (3d)

Yellow solid; Yield: $70 \%$; MP: $258-260^{\circ} \mathrm{C}$; IR $\left(v_{\max } \mathrm{cm}^{-1}\right)$ : 3369 , 3215, $3122(\mathrm{~N}-\mathrm{H}), 3084(=\mathrm{C}-\mathrm{H}), 1712(\mathrm{C}=\mathrm{O})$, 1612, 1566, 1492, $1481\left(\mathrm{C}=\mathrm{C}, \mathrm{NO}_{2}, \mathrm{~N}-\mathrm{H}, \mathrm{C}-\mathrm{N}\right), 1031(\mathrm{C}-\mathrm{Cl}), 844(=\mathrm{C}-\mathrm{H}) .{ }^{1} \mathrm{H}-\mathrm{NMR}$ (DMSO- $d_{6} / \mathrm{TMS}, 400 \mathrm{MHz}, \delta$ in $\left.\mathrm{ppm}\right): 7.78-8.68(\mathrm{~m}, 10 \mathrm{H}$, Ar-H), 9.43 (s, 1H, NH), 10.00 (s, 1H, NH), 10.75 (s, 2H, NH). $\mathrm{C}_{20} \mathrm{H}_{14} \mathrm{Cl}_{2} \mathrm{~N}_{6} \mathrm{O}_{5}$ (M.W.: $489.27 \mathrm{~g} / \mathrm{mol}$ ) calculated (\%): C:49.10, $\mathrm{H}: 2.88, \mathrm{~N}: 17.18$. Found: C:50.35, H:2.97, N:16.85.

1-(5-(2-(4-Methylbenzoyl)hydrazinecarbonyl)pyridin-2-yl)-3-(4nitrophenyl)urea (3e)

Yellow solid; Yield: $65 \%$; MP: $275-277^{\circ} \mathrm{C}$; IR $\left(v_{\max } \mathrm{cm}^{-1}\right)$ : 3369 , 3205, $3122(\mathrm{~N}-\mathrm{H}), 3049$ (=C-H stretching), $2987(\mathrm{C}-\mathrm{H}), 1712$ $(\mathrm{C}=\mathrm{O}), 1610,1562,1508,1492,1431$ (C=C, NO$, \mathrm{N}-\mathrm{H}, \mathrm{C}-\mathrm{N}), 844$ $(=\mathrm{C}-\mathrm{H})$. ${ }^{1} \mathrm{H}-\mathrm{NMR}$ (DMSO-d $/ \mathrm{TMS}, 400 \mathrm{MHz}, \delta$ in ppm): 2.39 (s, 3H, $\left.-\mathrm{CH}_{3}\right), 7.47-8.81(\mathrm{~m}, 11 \mathrm{H}, \mathrm{Ar}-\mathrm{H}), 9.45(\mathrm{~s}, 1 \mathrm{H}, \mathrm{NH}), 10.06$ (s, $1 \mathrm{H}, \mathrm{NH}$ ), 10.77 (s, $2 \mathrm{H}, \mathrm{NH}$ ). $\mathrm{C}_{21} \mathrm{H}_{18} \mathrm{~N}_{6} \mathrm{O}_{5}$ (M.W.: $434.41 \mathrm{~g} / \mathrm{mol}$ ) calculated (\%): C:58.06, H:4.98, N:19.15. Found: C:57.23, H:5.05, $\mathrm{N}: 18.56$.
1-(5-(2-(4-Bromobenzoyl)hydrazinecarbonyl)pyridin-2-yl)-3-(4nitrophenyl)urea (3f)

Yellow solid; Yield: $75 \%$; MP: $240-241^{\circ} \mathrm{C}$; IR $\left(v_{\max } \mathrm{cm}^{-1}\right)$ : 3207, 3122 $(\mathrm{N}-\mathrm{H}), 3082(=\mathrm{C}-\mathrm{H}$ stretching), $1712(\mathrm{C}=\mathrm{O}), 1610,1562,1508$, 1491, $1431\left(\mathrm{C}=\mathrm{C}, \mathrm{NO}_{2}, \mathrm{~N}-\mathrm{H}, \mathrm{C}-\mathrm{N}\right), 844(=\mathrm{C}-\mathrm{H}) .{ }^{1} \mathrm{H}-\mathrm{NMR}$ (DMSO- $d_{6} /$ TMS, $400 \mathrm{MHz}, \delta$ in ppm): 7.45-8.81 (m, 11H, Ar-H), $9.36(\mathrm{~s}, 1 \mathrm{H}$, $\mathrm{NH}$ ), 10.20 (s, 1H, NH), 10.84 (s, 2H, NH). $\mathrm{C}_{20} \mathrm{H}_{15} \mathrm{BrN}_{6} \mathrm{O}_{5}$ (M.W.: $499.27 \mathrm{~g} / \mathrm{mol}$ ) calculated (\%): C:48.11, H:3.03, N:16.83 Found: C:47.48, H:3.25, N:16.92.

1-(4-nitrophenyl)-3-(5-(2-(4-(trifluoromethyl)benzoyl) hydrazinecarbonyl)pyridin-2-yl)urea (3g)

Yellow solid; Yield: $70 \%$; MP: $299-300^{\circ} \mathrm{C}$; IR $\left(v_{\max } \mathrm{cm}^{-1}\right)$ : 3333 , 3271, $3213(\mathrm{~N}-\mathrm{H}), 3080(=\mathrm{C}-\mathrm{H}$ stretching), $1712(\mathrm{C}=\mathrm{O}), 1680$ ( $\mathrm{C}=\mathrm{O}$ hydrazide), $1614(\mathrm{C}=\mathrm{N}), 1564,1489,1431,1411\left(\mathrm{C}=\mathrm{C}, \mathrm{NO}_{2}\right.$, $\mathrm{N}-\mathrm{H}, \mathrm{C}-\mathrm{N}$ ), 1261 (C-F), $890(=\mathrm{C}-\mathrm{H}) .{ }^{1} \mathrm{H}-\mathrm{NMR}$ (DMSO- $d_{6} / \mathrm{TMS}$, $400 \mathrm{MHz}, \delta$ in ppm): 7.64-8.84 (m, 11H, Ar-H), $9.75(\mathrm{~s}, 1 \mathrm{H}, \mathrm{NH})$, $9.97(\mathrm{~s}, 1 \mathrm{H}, \mathrm{NH}), 10.72(\mathrm{~s}, 2 \mathrm{H}, \mathrm{NH}) . \mathrm{C}_{21} \mathrm{H}_{15} \mathrm{~F}_{3} \mathrm{~N}_{6} \mathrm{O}_{5}$ (M.W.: 488.38 $\mathrm{g} / \mathrm{mol}$ ) calculated (\%): C:51.65, H:3.10, N:17.21 Found: C:51.48, $\mathrm{H}: 3.25, \mathrm{~N}: 16.92$.

1-(5-(2-(4-methoxybenzoyl)hydrazinecarbonyl)pyridin-2-yl)-3(4-nitrophenyl)urea (3h)

Yellow solid; Yield: $75 \%$; MP: $222-223^{\circ} \mathrm{C}$; IR $\left(v_{\max } \mathrm{cm}^{-1}\right)$ : 3275 , 3171, $3117(\mathrm{~N}-\mathrm{H}), 3080$ (=C-H stretching), 2978 (C-H asymmetric stretching), 2841 (C-H symmetric stretching), 1703 (C=O), 1662 ( $\mathrm{C}=\mathrm{O}$ hydrazide), $1633(\mathrm{C}=\mathrm{N}), 1537,1506,1499,1471\left(\mathrm{C}=\mathrm{C}, \mathrm{NO}_{2}\right.$, $\mathrm{N}-\mathrm{H}, \mathrm{C}-\mathrm{N}$ ), 1327 (C-O), 824 (=C-H). ${ }^{1} \mathrm{H}-\mathrm{NMR}$ (DMSO-d $/$ /TMS, $400 \mathrm{MHz}, \delta$ in ppm): $3.84\left(\mathrm{~s}, 3 \mathrm{H}, \mathrm{OCH}_{3}\right), 7.05-8.84(\mathrm{~m}, 11 \mathrm{H}$, Ar-H), 9.79 (2s, 1H, NH), 10.45 (2s, 1H, NH), 10.72 (s, 2H, NH). $\mathrm{C}_{21} \mathrm{H}_{18} \mathrm{~N}_{6} \mathrm{O}_{6}$ (M.W.: $450.40 \mathrm{~g} / \mathrm{mol}$ ) calculated (\%): C:56.00, H:4.03, $\mathrm{N}: 18.66$ Found: C:56.48, $\mathrm{H}: 3.25, \mathrm{~N}: 18.92$.

1-(5-(2-benzoylhydrazinecarbonyl)pyridin-2-yl)-3-(4nitrophenyl)urea (3i)

Yellow solid; Yield: $70 \%$; MP: 203-204 ${ }^{\circ} \mathrm{C}$; IR $\left(v_{\max } \mathrm{cm}^{-1}\right)$ : 3271 , 3213, $3124(\mathrm{~N}-\mathrm{H}), 3045(=\mathrm{C}-\mathrm{H}$ stretching), $1712(\mathrm{C}=\mathrm{O}), 1666$ (C=O hydrazide), 1641 ( $C=N), 1602,1562,1506,1489,1431$ ( $C=C$, $\mathrm{NO}_{2}, \mathrm{~N}-\mathrm{H}, \mathrm{C}-\mathrm{N}$ ), 846 (=C-H). ${ }^{1} \mathrm{H}-\mathrm{NMR}$ (DMSO- $d_{6} / \mathrm{TMS}, 400 \mathrm{MHz}$, $\delta$ in ppm): 7.45-8.84 (m, 11H, Ar-H), $9.80(2 \mathrm{~s}, 1 \mathrm{H}, \mathrm{NH}), 10.68$ (2s, $1 \mathrm{H}, \mathrm{NH}), 11.48(\mathrm{~s}, 2 \mathrm{H}, \mathrm{NH}) . \mathrm{C}_{20} \mathrm{H}_{16} \mathrm{~N}_{6} \mathrm{O}_{5}(\mathrm{M} . \mathrm{W} .: 420.38 \mathrm{~g} / \mathrm{mol})$ calculated (\%): C:57,14 H:3.84, N:19.99 Found: C:57.48, H:3.65, $\mathrm{N}: 19.92$.

1-(4-nitrophenyl)-3-(5-(2-(4-(trifluoromethylthio)benzoyl) hydrazinecarbonyl)pyridin-2-yl)urea (3j)

Yellow solid; Yield: 65\%; MP: $277-278^{\circ} \mathrm{C}$; IR $\left(v_{\max } \mathrm{cm}^{-1}\right): 3333$, $3221(\mathrm{~N}-\mathrm{H}), 3080(=\mathrm{C}-\mathrm{H}$ stretching), 1714 ( $\mathrm{C}=\mathrm{O}$ urea), $1680(\mathrm{C}=\mathrm{O}$ hydrazide), $1641(\mathrm{C}=\mathrm{N}), 1564,1510,1492\left(\mathrm{C}=\mathrm{C}, \mathrm{NO}_{2}, \mathrm{~N}-\mathrm{H}, \mathrm{C}-\mathrm{N}\right)$, $844(=\mathrm{C}-\mathrm{H})$. ${ }^{1} \mathrm{H}-\mathrm{NMR}$ (DMSO- $d_{6} / \mathrm{TMS}, 400 \mathrm{MHz}$, $\delta$ in ppm): 7.63$8.83(\mathrm{~m}, 11 \mathrm{H}, \mathrm{Ar}-\mathrm{H}), 9.79$ (2s, 1H, NH), 9.97 (s, 1H, NH), 10.72 (s, $2 \mathrm{H}, \mathrm{NH}$ ). $\mathrm{C}_{21} \mathrm{H}_{15} \mathrm{~F}_{3} \mathrm{~N}_{6} \mathrm{O}_{5} \mathrm{~S}$ (M.W.: $520.44 \mathrm{~g} / \mathrm{mol}$ ) calculated (\%): C:48.46, H:2.91, N:16.15 Found: C:48.76, H:3.05, N:16.92.

\section{Biology}

\section{Cell culture}

A human pancreatic adenocarcinoma Capan1 cell line, human cervix carcinoma HeLa cell line, human lung fibroblasts MRC5 
cell line, human breast adenocarcinoma MCF7 and HCC1937 cell line were obtained from American Type Culture Collection (Bethesda), and maintained as exponentially growing monolayers by culturing according to the supplier's instructions in a humidifier incubator at $37^{\circ} \mathrm{C}$ supplied with $5 \% \mathrm{CO}_{2}$. All cell culture reagents were purchased from Biological Industries (Israel).

\section{Cytotoxicity test}

The cytotoxic potencies of the test compounds were determined using a WST1 Cell Protliferation Assay (Roche) according to the manufacturer's instructions. All tested compounds were dissolved in DMSO. Cells were seeded into a 96-well plate at a density of 5000 cells/well for HeLa, MCF7 and HCC1937, 7500 cells/well for Capan1 and 10,000 cells per well for MRC5. The next day, cells were treated with compounds with the final concentrations of $1,2,5,10,25 \mu \mathrm{M}$ and incubated for 48 hours at conventional cell culture conditions. DMSO was used a negative solvent control, and doxorubicin was included in the study as a positive cytotoxic control compound. The ratio of surviving cells after compound treatment was determined using a colorimetric WST-1 assay (Roche) as indicated in the protocol provided by manufacturer. The absorbance was measured using a Varioscan microplate reader (Thermo) at 450 $\mathrm{nm}$ with a 620-nm reference filter. To determine the $\mathrm{IC}_{50}$ values, a sigmoid-dose response curve was fitted to the data using nonlinear regression on GraphPad Prism 5 software.

This study did not need ethics committee approval because in vitro methods were used for biologic activity processes.

\section{RESULTS AND DISCUSSION}

The synthetic route to the target compounds is outlined in Scheme 2. The structures of the compounds (1, 2, 3a-j) were confirmed using IR, ${ }^{1} \mathrm{H}-\mathrm{NMR}$, and elemental analysis. IR spectra of the compounds (1, 2, 3a-j) afforded N-H stretching (3115-3369) bands. IR spectra of all compounds (1, 2, 3a-j) were described $\mathrm{C}-\mathrm{H}$ stretching (3045-3082), urea and carbohydrazide $\mathrm{C}=\mathrm{O}$ stretching (1662-1714) bands, aromatic rings $\mathrm{C}=\mathrm{C}$ stretching and $\mathrm{NO}_{2}$ stretching (1411-1604) bands. The $\mathrm{NH}$ protons of carbohydrazide and urea groups resonated as two different singlet peak at 9.36-11.48 ppm. The aromatic protons displayed a multiplet at 7.05-8.85 ppm. The elemental analysis of compounds was in agreement with the proposed structures of the compounds.

It is known that some PARP inhibitors are highly-selective promising agents against cancer cells with homologous recombination (HR) DNA repair pathway deficiencies such as those harboring mutations on tumor suppressors BRCA1 or $B R C A 2$ via generation of chromatid breaks, cell cycle arrest and apoptosis. 16,17 Therefore, we included HCC1937 and Capan1, which are defective in BRCA1 and BRCA2, respectively. We aimed to compare the cytotoxicity of the compounds on these cell lines with their activities on HR-proficient cancer cell lines (HeLa and MCF7) and also a non-tumoral MRC5 fibroblast cell line.

When the substitution pattern at the phenyl ring was determined, the effect of electron donor and electron acceptor groups on activity was considered. Therefore, methyl, methoxy, and halogens such as $\mathrm{F}, \mathrm{Cl}, \mathrm{Br}$ were selected as electron donors and a nitro group was selected as an electron acceptor. Our data suggest that only $3 a$ having fluoro, $3 b$ having chloro, $3 c$ having nitro and $3 d$ having 2,6 dichloro substituents had cytotoxic activities at the tested concentrations. The $\mathrm{IC}_{50}$ values of these compounds are given in Table 1. Compounds $3 e-j$ showed less cytotoxic activity on all cancer cells compared with 3a-d. However, our results suggest that the compounds possessed no selectivity toward HR defective Capan1 and HCC1937 cells harboring BRCA mutations compared with MCF7 and HeLa cells with intact HR pathways.

\section{CONCLUSIONS}

In the present paper, we reported the synthesis of some new urea and carbohydrazide derivatives from methyl 6-aminopyridine-

Table 1. Cytotoxic activity of compounds

$$
\mathrm{IC}_{50}(\mu \mathrm{M})
$$

\begin{tabular}{|c|c|c|c|c|c|}
\hline Compounds & HCC1937 & Capan1 & MCF7 & HeLa & MRC5 \\
\hline $3 a$ & $7.6 \pm 0.09$ & $7.4 \pm 0.62$ & $7.3 \pm 0.86$ & $6.6 \pm 0.53$ & $15.4 \pm 1.42$ \\
\hline $3 b$ & $8.9 \pm 0.07$ & $8.4 \pm 0.26$ & $9.2 \pm 0.49$ & $11.7 \pm 1.02$ & $19.6 \pm 1.06$ \\
\hline $3 c$ & $10.4 \pm 0.6$ & $9.3 \pm 0.79$ & $9.6 \pm 0.56$ & $9.8 \pm 1.86$ & $18.3 \pm 1.54$ \\
\hline $3 d$ & $7.8 \pm 0.82$ & $7.3 \pm 0.75$ & $7.5 \pm 0.13$ & $7.9 \pm 1.68$ & $17.4 \pm 1.12$ \\
\hline $3 e$ & $>25$ & $>25$ & $>25$ & $>25$ & $>25$ \\
\hline $3 f$ & $>25$ & $>25$ & $>25$ & $>25$ & $>25$ \\
\hline $3 g$ & $>25$ & $>25$ & $>25$ & $>25$ & $>25$ \\
\hline $3 \mathrm{~h}$ & $>25$ & $>25$ & $>25$ & $>25$ & $>25$ \\
\hline $3 i$ & $>25$ & $>25$ & $>25$ & $>25$ & $>25$ \\
\hline $3 j$ & $>25$ & $>25$ & $>25$ & $>25$ & $>25$ \\
\hline Doxorubicin & $1.05 \pm 0.07$ & $0.98 \pm 0.08$ & $1.13 \pm 0.12$ & $0.73 \pm 0.13$ & $7.2 \pm 1.37$ \\
\hline
\end{tabular}


3-carboxylate. The synthesized compounds were evaluated for their cytotoxic activity. Our data indicate that 3a-d are more selective to cancer cells compared with nontumoral fibroblasts; however, these compounds are not more potent on HR defective cells with BRCA mutants.

\section{ACKNOWLEDGEMENTS}

This study was supported by TÜBITAK 215 S112 and Marmara University Scientific Research Commission (project number: SAG-C-DRP-100616-0260).

Conflict of Interest: No conflict of interest was declared by the authors.

\section{REFERENCES}

1. Nepali K, Sharma S, Sharma M, Bedi PM, Dhar KL. Rational approaches, design strategies, structure activity relationship and mechanistic insights for anticancer hybrids. Eur J Med Chem. 2014;77:422-487.

2. Liu Z, Wang $Y$, Lin $H$, Zuo D, Wang L, Zhao Y, Gong P. Design, synthesis and biological evaluation of novel thieno[3,2-d]pyrimidine derivatives containing diaryl urea moiety as potent antitumor agents. Eur $\mathrm{J}$ Med Chem. 2014:85:215-227.

3. Horowitz S, Trievel RC. Carbon-oxygen hydrogen bonding in biological structure and function. J Biol Chem. 2012;287:41576-41582.

4. He H, Wang X, Shi L, Yin W, Yang Z, He H, Liang Y. Synthesis, antitumor activity and mechanism of action of novel 1,3-thiazole derivatives containing hydrazide-hydrazone and carboxamide moiety. Bioorg Med Chem Lett. 2016;26:3263-3270.

5. Morjan RY, Mkadmh AM, Beadham I, Elmanama AA, Mattar MR, Raftery J, Pritchard RG, Awadallah AM, Gardiner JM. Antibacterial activities of novel nicotinic acid hydrazides and their conversion into $\mathrm{N}$-acetyl-1,3,4oxadiazoles. Bioorg Med Chem Lett. 2014;24:5796-5800.

6. Altıntop MD, Özdemir A, Turan-Zitouni G, Ilgın S, Atlı Ö, İșcan G, Kaplancıklı ZA. Synthesis and biological evaluation of some hydrazone derivatives as new anticandidal and anticancer agents. Eur J Med Chem. 2012;58:299-307.

7. Salgin-Gökșen U, Gökhan-Kelekçi N, Göktaș O, Köysal Y, Kiliç E, Ișik S, Aktay G, Ozalp M. 1-Acylthiosemicarbazides, 1,2,4-triazole-5(4H)thiones, 1,3,4-thiadiazoles and hydrazones containing 5-methyl- 2-benzoxazolinones: Synthesis, analgesic-anti-inflammatory and antimicrobial activities. Bioorg Med Chem. 2007;15:5738-5751.

8. Chen JN, Wang XF, Li T, Wu DW, Fu XB, Zhang GJ, Shen XC, Wang HS. Design, synthesis and biological evaluation of novel quinazolinyldiaryl urea derivatives as potential anticancer agents. Eur J Med Chem. 2015;107:12-25.

9. Wang $W$, Kong $D$, Cheng $H$, Tan L, Zhang Z, Zhuang $X$, Long $H$, Zhou $Y$, $X u Y$, Yang $X$, Ding K. New benzimidazole-2-urea derivatives as tubulin inhibitors. Bioorg Med Chem Lett. 2014;24:4250-4253.

10. Fortin S, Moreau E, Lacroix J, Cote MF, Petitclerc E, Gaudreault R. Synthesis, antiproliferative activity evaluation and structure-activity relationships of novel aromatic urea and amide analogues of $\mathrm{N}$-phenylN'-(2-chloroethyl)ureas. Eur J Med Chem. 2010;45:2928-2937.

11. De P, Baltas M, Lamoral-Theys D, Bruyere C, Kiss R, BedosBelval F, Saffon N. Synthesis and anticancer activity evaluation of 2-(4-alkoxyphenyl)cyclopropyl hydrazides and triazolo phthalazines. Bioorg Med Chem. 2010;18:2537-2548.

12. Kurumurthy C, Veeraswamy B, Sambasiva Rao P, Santhosh Kumar G, Shanthan Rao P, Loka Reddy V, Venkateswara Rao J, Narsaiah B. Synthesis of novel 1,2,3-triazole tagged pyrazolo[3,4-b]pyridine derivatives and their cytotoxic activity. Bioorg Med Chem Lett. 2014;24:746-749.

13. Ghorab MM, Alqasoumi SI, Abdel-Kader MS, Alsaid MS. Utility of L-Norephedrine in the semisynthesis of novel thiourea and thiazolidine derivatives as a new class of anticancer agents. Acta Pol Pharm. 2014;71:615-623.

14. Jha KK, Samad A, Kumar Y, Shaharyar M, Khosa RL, Jain J, Kumar V, Singh P. Design, synthesis and biological evaluation of 1,3,4-oxadiazole derivatives. Eur J Med Chem. 2010;45:4963-4967.

15. Lu C, Tang K, Li Y, Li P, Lin Z, Yin D, Chen X, Huang H. Design, synthesis and evaluation of novel diaryl urea derivatives as potential antitumor agents. Eur J Med Chem. 2014;77:351-360.

16. Bryant HE, Schultz N, Thomas HD, Parker KM, Flower D, Lopez E, Kyle S, Meuth M, Curtin NJ, Helleday T. Specific killing of BRCA2-deficient tumours with inhibitors of poly(ADP-ribose) polymerase. Nature. 2005;434:913-917.

17. Farmer $H, M c C a b e ~ N$, Lord CJ, Tutt AN, Johnson DA, Richardson TB, Santarosa M, Dillon KJ, Hickson I, Knights C, Martin NM, Jackson SP, Smith GC, Ashworth A. Targeting the DNA repair defect in BRCA mutant cells as a therapeutic strategy. Nature. 2005;434:917-921. 